\title{
PHOTOCURRENT AND INTRINSIC MODULATION SPEEDS IN P-I(MQW)-N GaAs/AIGaAs STARK EFFECT MODULATORS
}

\author{
S. Hong*, J. Loehr, S. Goswami, P. Bhattacharya and J. Singh \\ Department of Electrical Engineering and Computer Science \\ The University of Michigan, Ann Arbor, MI 48109-2122 USA
}

(Received 30 July 1990)

\begin{abstract}
Theoretical and experimental studies on photocurrent are presented in p-i-n $\mathrm{GaAs} / \mathrm{Al}_{0.3} \mathrm{Ga}_{0.7} \mathrm{As} \mathrm{MQW}$ modulators and detectors. Field dependent excitonic spectra is calculated to obtain carrier generation and recombination rates using full band mixing effects in the valence band. A tunneling formalism is used to self consistently study the electric field dependence of the electron and hole collection efficiency. At low electric fields and low temperatures the carrier collection efficiency is very poor and we show that the collected photocurrent has a sublinear dependence on the impinging light intensity. This non-linearity is not due to band filling or exciton screening effects normally observed, but arises from the high recombination rates compared to tunneling rates. At higher electric field the collection efficiency is $\sim 100 \%$ resulting in a linear response and a negative differential resistance region. Consequences of our studies on intrinsic device speeds are discussed for $\mathrm{p}-\mathrm{i}(\mathrm{MQW})-\mathrm{n}$ modulators based on Stark effect.
\end{abstract}

\section{Introduction}

The quantum confined Stark effect (QCSE) [1-4] can be exploited for optical modulators as well as for opto-electronic devices which rely on the negative resistance in the photocurrent voltage relation. The negative resistance arises in a $\mathrm{p}-\mathrm{i}(\mathrm{MQW})-\mathrm{n}$ structure because of the shift of the heavy hole excitonic peak and the dip in absorption coefficient between the heavy hole (HH) and light hole (LH) excitonic peaks.

Both modulation devices as well as photocurrent based switching devices depend critically upon the photocurrent. The modulation device's intrinsic speed is dependent upon how fast the e-h pairs created by absorption can be removed from the MQW region. The photocurrent in the $\mathrm{p}-\mathrm{i}-(\mathrm{MQW})-\mathrm{n}$ structure involves: i) carrier generation by light absorption, ii) carrier recombination leading to luminescence and iii) carrier collection via tunneling or thermionic emission and drift. To understand the photocurrent we have developed a self-consistent formalism to address the three processes described above. We have also carried out detailed experimental studies to study the temperature and bias

-Present address: Korea Institute of Technology, Department of Electrical Engineering and Computer Science, Taejon, 305-338, KOREA. dependence of the photocurrent in GaAs/AlGaAs MQW structures.

In the next section, our theoretical formalism is outlined along with key results. In Sec. III, the experimental results are presented. We conclude in Sec. IV.

\section{Theoretical Considerations}

Several important aspects are involved in the photocurrent phenomenon. These are: i) optical absorption (excitonic and/or band to band) in the quantum wells in the presence of electric field; ii) radiative recombination of electron-hole pairs through excitonic or band to band states - this is responsible for photoluminescence; iii) non-radiative recombination of the e-h pairs; and iv) tunneling/emission of the electrons and holes resulting in photocurrent. It is important to understand all these phenomena as a function of optical intensity, temperature, electric field and the nature of the quantum well (square, asymmetric etc). We will assume low input optical power so that only linear optical absorption region is considered, but in this regime all the phenomena described above need not be linear. Due to space limitations, the details of our formalism will be presented elsewhere.

A number of groups have calculated the field depen- 
dent excitonic and band to band absorption spectra for the quantum well structures [6-8].

Once the optical absorption is calculated, it is straightforward to calculate the radiative recombination rates for the e-h pairs. It is assumed that there is no stimulated emission taking place since the photon intensity is quite low. The recombination rate is given by

$$
U=\int \frac{f e\left(E_{e}\right) f_{h}\left(E_{h}\right) D(E)}{\tau_{\text {eh }}(E)} d E
$$

where $f_{c}\left(E_{s}\right)$ and $f_{h}\left(E_{h}\right)$ are the Fermi-Dirac distribution functions for elections and holes, and $D(E)$ is a joint density of states of the electrons and holes, $\tau_{a h}$ is the electron and hole recombination time. This recombination time $r_{e h}$ can be calculated from the absorption coefficient

$$
\frac{1}{\tau_{\text {eh }}(E)}=\frac{\alpha(E) v D_{p h}(E)}{D(E)}
$$

where $D_{p h}$ is the density of photon states, $v$ velocity of light, and $\alpha(E)$ the absorption coefficient.

The final ingredient in calculating the photocurrent is the tunneling (both quantum mechanical and phonon assisted i.e. below and above the barrier) of the electrons and holes. A formalism for the tunneling process is described in reference 9.

Focussing on the carrier density in the $i^{\text {th }}$ single quantum well one can assume the carrier density is dependent on i) the generation rates $\left(G_{e}, G_{h}\right)$, ii) recombination rates $\left(\mathrm{U}_{e}, \mathrm{U}_{h}\right)$ and iii) average tunneling-out rates $\left(T_{c}, T_{h}\right)$ of electrons and holes in the quantum well. The rate equations which describe the carrier density can be written as:

$$
\begin{aligned}
& \frac{\partial n}{\partial t}=G_{e}-U_{c}-\bar{T}_{c} \\
& \frac{\partial p}{\partial t}=G_{h}-U_{h}-\bar{T}_{h}
\end{aligned}
$$

For the p-i-n structure considered, the generation rates and recombination rates must be the same respectively $\left(G_{c}=G_{h}, U_{d}=U_{h}\right)$ for the steady state, and therefore $\bar{T}_{a}$ should be equal to $\bar{T}_{h}$. The generation rates of electron and hole can be described by $\mathrm{I}_{o p} \alpha(E) \mathrm{L}$, where $\mathrm{I}_{o p}$ is the photon flux density, $\alpha(E)$ is the absorption coefficient at the energy $E$, and $L$ is the well width. The average tunneling-out term can be calculated from the difference of the tunneling-in rate and tunneling-out rate. The sequential tunneling through a finite number quantum well is neglected because in MQW structures this effect is very small compared to that of single barrier tunneling. These average tunneling rates are then given by

$$
T_{\varepsilon}=\int_{E_{B}^{s}}^{E_{g}^{s}} T_{e}(E) f_{e}(E, \phi) g_{e}(E) d E
$$

$$
\bar{T}_{h}=\int_{E_{o}^{k}}^{E_{B}^{k}} T_{h}(E) f_{\Lambda}(E, \phi) g_{h}(E) d E
$$

where $\phi_{e}, \phi_{h}$ are quasi Fermi level for electrons and holes respectively, and $g_{e}(E), g_{h}(E)$ is the two dimensional electron and hole density of states.

A lower limit for the tunneling of the electrons is introduced since the probability of tunneling through more than one barrier is extremely small. The upper limit for the tunneling (thermionic emission process) is introduced since beyond this limit the electrons can be emitted equally towards the $i-1$ and $i+1$ wells. As can be expected the dominant contribution to the current comes from carriers very close to the barrier heights. For our calculations, the tunneling rates are obtained by the WKB method.

As seen from the rate equations, electron tunneling current should be the same as hole tunnelling current. Thus the calculations have to be done self consistently to reach this balance and thus the steady state properties. The transit time is found to be much smaller than tunneling out time in $1 \sim 2 \mu \mathrm{m} \mathrm{MQW} \mathrm{structures,}$ and thus the transport to the $p-i-n$ diode electrodes is primarily determined by tunneling mechanism of electrons and holes. Neglecting the current components which are from $n^{+} p^{+}$depletion region absorption and minority carrier diffusion from bulk to depletion region, one can write the photocurrent as approximately

$$
J=2 N \bar{T}_{e}
$$

where $N$ is the number of quantum wells.

By solving the rate equations with respect to quasiFermi levels $\phi_{e}, \phi_{h}$ self-consistently we obtain the various opto-electronic properties of the structure.

Before presenting our calculated results for the photocurrent spectra, steady state carrier densities, recombination rates etc. we physically examine two extreme limits. On one extreme we have a situation where the applied field (external field and built in field) is very small so that the tunneling out rates are very close to zero. In this case the recombination processes dominate and we have the relation

$$
G \propto I_{o p} \cong U \propto n \cdot p
$$

i.e. the steady state electron and hole densities are proportional to $I_{o \sigma}^{1 / 2}$. Since the tunneling current is proportional to the carrier densities we expect that in this regime

$$
J \alpha I_{o p}^{1 / 2}
$$

Obviously, in this region the quantum efficiency would be very small. Notice that this non-linear behavior has nothing to do with the usual non-linear op- 
tical absorption effects related to high intensity $(\geq 1$ $\mathrm{KW} / \mathrm{cm}^{2}$ ), but is simply due to the carrier densities being dominated by recombination proceseses leading to a non-linear current response.

On the other extreme we have the situation where the field is very high so that the tunneling out processes are very fast so that essentially all the generated carriers are collected and recombination processes are not significant. In this regime

$$
G \alpha I_{0} \cong T_{.}
$$

and we expect a linear response with essentially $100 \%$ quantum efficiency. It is important to establish quantitatively in which range of parameters one expects the non-linear response since this may be of importance in optically addressed modulators.

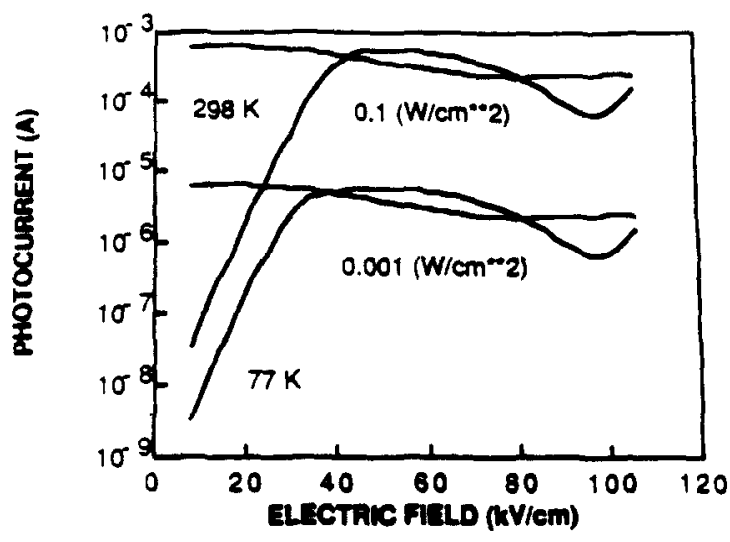

Figure 1

Effect of optical intensity on photocurrent at room temperature and $77 \mathrm{~K}$.

In Fig. 1, we show the calculated intensity dependence of photocurrent vs. electric field results at room temperature and $77 \mathrm{~K}$ for $\mathrm{a} p-\mathrm{i}-\mathrm{n}$ diode with area 3 $\times 10^{-3} \mathrm{~cm}^{2}$ with 50 periods of $100 \mathrm{~A}$ well and barrier. The input optical power is $0.001 \mathrm{watt} / \mathrm{cm}^{2}$ and the input frequency is chosen to maximize the negative resistance region of the $I-V$ characteristics. This involves chosing the photon energy slightly smaller than the zero bias HH exciton energy. For the results reported, the laser energy was chosen to be $1.460 \mathrm{eV}$ and $1.544 \mathrm{eV}$ at room temperatures and $77 \mathrm{~K}$.

Several important observations can be made from the results of Fig. 1. At low temperatures we note that even at fairly high bias values the photocurrent is extremely small. Here the e-h pairs that are created by absorption recombine emitting photons and do not lead to any significant current. The quantum
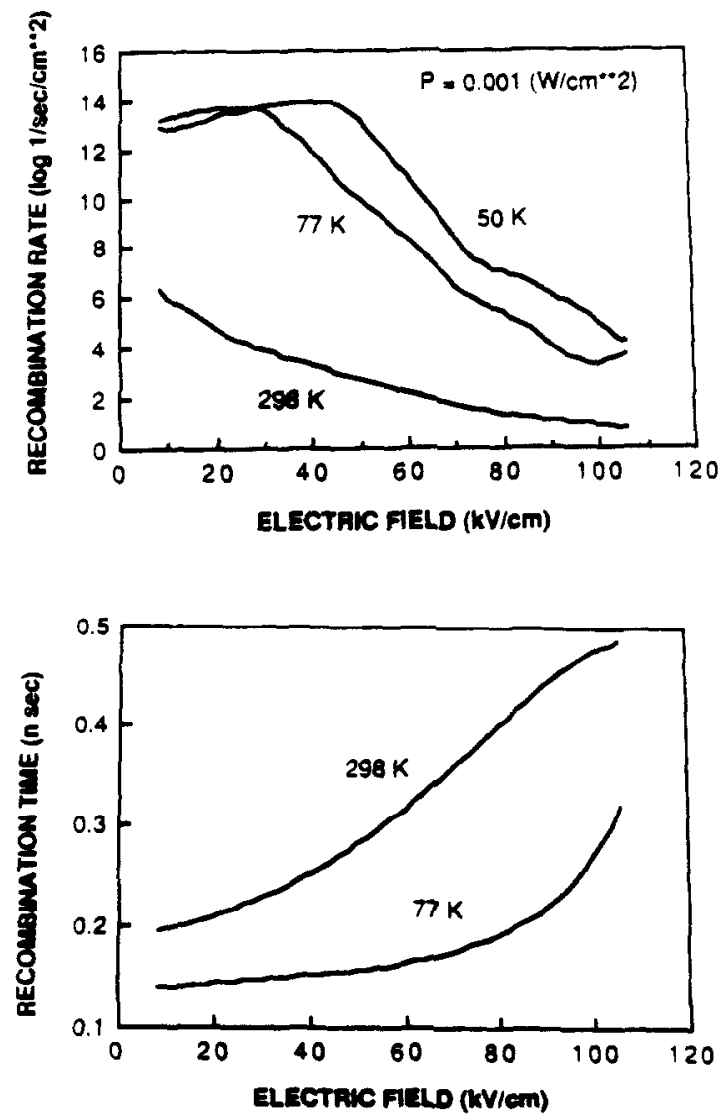

Figure 2

a) Radiative recombination rates at different temperatures in a $1 \mathrm{\mu m}$ thick GaAs/AlGaAs (100 A well and barrier) MQW structure; b) Average recombination time at $298 \mathrm{~K}$ and $77 \mathrm{~K}$ as a function of electric field.

efficiency is rather poor and the steady state properties of the p-i(MQW)-n structure are controlled by the recombination processes. In this region we expect the photocurrent to have the non-linear dependence upon the optical power as discussed above. At room temperature, this region is not present since even at small applied biases, the e-h pairs can be controlled quite efficiently. At low temperatures once the applied bian is high, the tunneling out terms dominate and one gets a situation where all the e-h pairs are collected.

In Fig. 2(a) we examine the field dependence of the recombination ratea at various temperatures. Note that even though the input power level is fixed, the generation rate is not fixed since the absorption spectra is field dependent.

At low temperatures and low fields we see that the recombination rate is essentially the same as the gener- 
ation rate. The slight increase with field seen is due to the above mentioned increase in generation rate. When the field is increased, the recombination rate drops by nearly seven orders of magnitude and in this regime all the e-h pairs generated lead to photocurrent. At room temperature, the recombination rates are $\sim$ seven orders of magnitude smaller at low field when compared to the low temperature case showing the dominance of the tunneling out rates.

In Fig. 2(b) we show the average e-h recombination time as a function of applied field. As the field is increased, the time increases, as expected, because of the separation of the e-h wave functions. The larger recombination time at higher temperature is due to the spread in the Fermi function and the consequent reduction in the probability of an electron to find a hole.

Thus, by examing the optical power dependence of the photocurrent, it is possible to predict the speed of the device. In the strictly linear dependence region, the tunneling out time is $\sim 1$ ps and since the transit time is $\sim 10 \mathrm{ps}$, the device can operate very fast. However, when the response is sublinear, the recombination terms dominate and the response is $\sim 0.5-1.0$ ns as expected from Fig. 3(b).

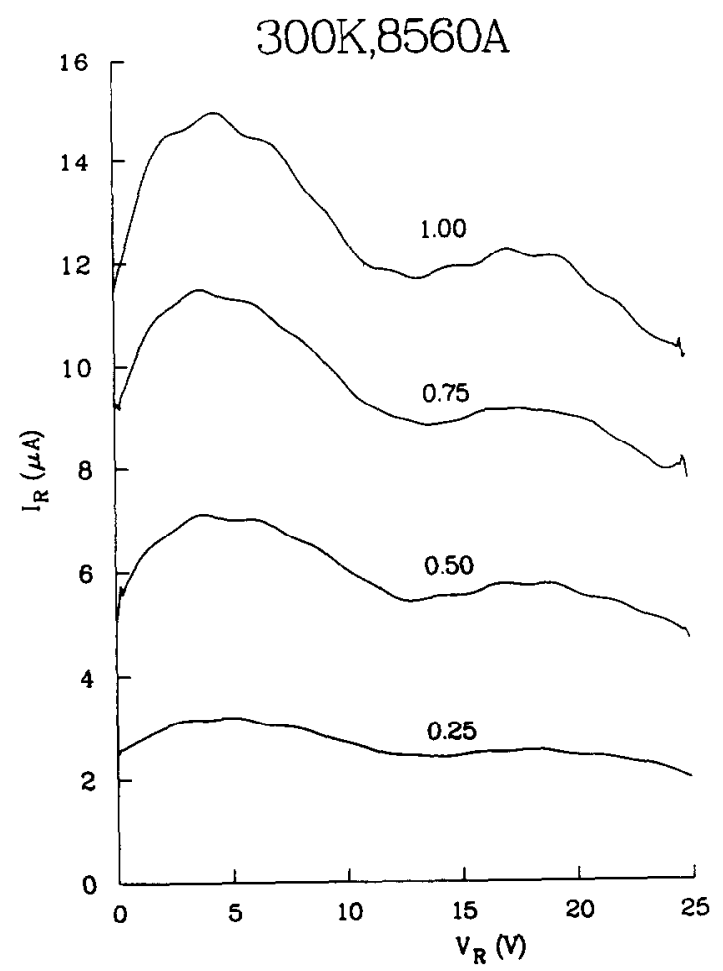

Figure 3

Measured photocurrent as a function of voltage at various optical intensity levels at $300 \mathrm{~K}$.

\section{Experimental Results}

P-i-n photodiodes for our experiments were grown by molecular beam epitaxy (MBE). The i-region consists of 100 periods of multiquantum well consisting of alternate layers of $100 \AA \mathrm{GaAs}$ and $100 \AA \mathrm{Al}_{0.3} \mathrm{Ga}_{0.7} \mathrm{As}$. $500-\mu \mathrm{m}$ diameter mesa diodes are defined by photolithography and appropriate ohmic contacts are formed on the $p$ - and $n$-sides by evaporation and annealing. The photocurrent for different values of reverse bias, excitation wavelength, excitation intensity and temperature was measured with a tunable dye laser, hiquid He cryostat and a HP 4145 parameter analyzer.

In Fig. 3 we show typical photocurrent-voltage data at room temperature. The wavelength chosen allows us to obtain strong negative resistance. Also shown are the intensity dependence of the photocurrent. We find that at room temperature, the photocurrent has a linear dependence on optical intensity. As discussed in the previous section this implies that tunneling and emission components dominate over recombination processes at this temperature. In Fig. 4 show the photocurrent versus optical intensity results for various bias conditions at $14 \mathrm{~K}$. This figure is displayed to bring out the non-linear behavior of the photocurrent more clearly. As can be seen from this figure, at low fields, the photocurrent is strongly sublinear $\left(\mathrm{I}_{p h} \alpha I_{o p}^{0.78}\right)$. At the high-

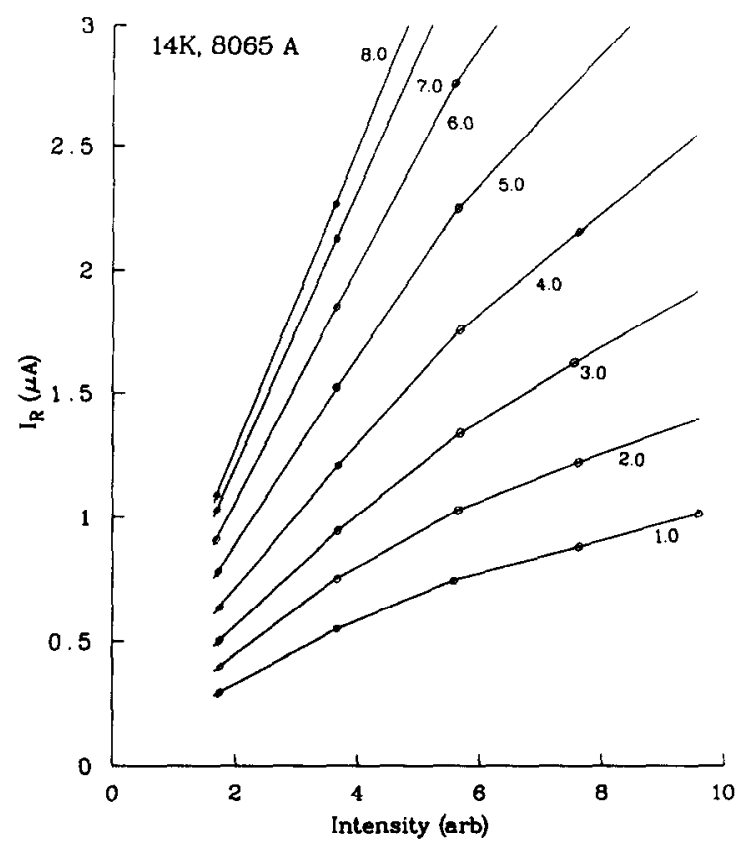

Figure 4

Intensity dependence of photocurrent at different applied bias values at $14 \mathrm{~K}$. 
est field considered, $(E \sim 80 \mathrm{kV} / \mathrm{cm})$, the photocurrent shows an essential linear dependence. These features are understood on the basis of the results of Sec. II.

Once again we note that the simple experiment observing the intensity dependence of the photocurrent tells us that if the response is perfectly linear, the recombination process is not controlling the removal of the e-h pairs and the device speeds are not limited by the recombination times as is often believed for these structures.

\section{Conclusions}

In this paper we have theoretically and experimentally examined the photocurrent and generation/ recombination processes in MQW structures. Photocurrent is important for use of these structures as modulators (e.g. the modulation speed depends upon the speed at which carriers can be extracted from the MQW structure), negative resistance based optical switches and detectors. Our study shows a number of interesting features. At low electric fields and low temperatures, the carrier extraction efficiency is very poor so that most carriers recombine providing efficient luminescence. Since the recombination time is slow $(\sim 0.5$ ns), modulators operating in this regime will be limited by this time. Non-linear response is also expected in this regime. On the other hand, at high temperatures and/or high electric fields, the tunneling efficiency improves and the intrinsic device speed will be limited by transit time effects as in bulk p-i-n detectors. We have also discussed the optical power dependence of the photocurrent and the carrier density for both electrons and holes. The non-linear regime discussed by us here could be of importance for certain optically addressed devices, although as discussed, the response time in this regime is slow (nanosecond range). We note that our results agree well with our experimental data on photocurrent behavior and other reported data on absorption, recombination times and photocurrent, giving us confidence in the formalism and the approximations made.

Acknowledgements - This work was supported by U.S. Air Force Office of Scientific Research (AFOSR88-0168).

\section{References:}

1. T. H. Wood, C. A. Burrus, D.A.B. Miller, D. S. Chemla, T. C. Damen, A. C. Gossard and W. Wiegmann, Applied Physics Letter Vol. 44, pp. 16-18 (1984).

2. D.A.B. Miller, D. S. Chemla, T. C. Damen, T. H. Wood, C. A. Burrus, A. C. Gossard and W. Wiegmann, IEEE Journal of Quantum Electronics QE-21, No. 9, pp. 1462-1476 (1985).

3. P. Wheatley, P. J. Bradley, M. Whitehead, G. Parry, J. E. Midwinter, P. Mistry, M. A. Pate and J. W. Roberts, Electronics Letter Vol. 23, pp. 92-93 (1985).

4. W. Li, S. Hong, J. Oh, J. Singh and P. Bhattacharya, Electronics Letter Vol. 25, pp. 476-477 (1989).

5. G. D. Sanders and Y. C. Chang, Physical Review $B$ Vol. 32, pp. 5517-5520 (1985).

6. S. Hong, M. Jaffe and J. Singh, IEEE Journal of Quantum Electronica QE-23, pp. 2181-2195 (1987).

7. S. Hong and J. Singh, Superlattice and Micrastructure, Vol. 3, pp. 645-656 (1987).

8. G. D. Sanders and K. K. Bajaj, Physical Review $B$ Vol. 35, (1987).

9. A. Larson, P. A. Andrekson, S. T. Eng and A. Yariv, IEEE Journal of Quantum Electronics QE24, pp. 787 (1988). 